\title{
Minimum-time constrained velocity planning ${ }^{\dagger}$
}

\author{
Gabriele Lini ${ }^{1}$, Luca Consolini, Aurelio Piazzi \\ Università di Parma \\ Dipartimento di Ingegneria dell'Informazione \\ Viale G.P. Usberti 181A \\ 43100 Parma, Italy \\ Email: gabriele.lini@gmail.com, luca.consolini@polirone.mn.it, aurelio.piazzi@unipr.it
}

\begin{abstract}
This paper proposes a method for minimum-time velocity planning with velocity, acceleration and jerk constraints and generic initial and final boundary conditions for the velocity and the acceleration. This minimum-time planning problem is relevant in the context of robotic autonomous navigation, where the iterative steering supervisor periodically replans the future mobile robot motion starting from current position, velocity and acceleration conditions. The problem is faced through discretization and its solution is based on a sequence of linear programming feasibility checks, depending on motion constraints and boundary conditions.
\end{abstract}

\section{INTRODUCTION}

The problem of motion planning for autonomous guided vehicles is a well known and studied issue in robotics, see for example the recent books [1] and [2]. This paper proposes a technique for minimum-time velocity planning, considering given jerk, acceleration and velocity constraints. This minimum-time planning problem is cast in the context of the so-called path-velocity decomposition [3] using the iterative steering navigation technique [4], [5]. In this scenery, it is important to plan a smooth velocity profile with arbitrary velocity and acceleration boundary conditions. The relevant velocity planning problem is the synthesis of a velocity $C^{1}$-function that permits in minimum-time, with bounded velocity, acceleration and jerk, to interpolate given initial and final conditions.

The minimum-time transition is obtained by discretizing the continuous-time model and formulating an equivalent discrete-time optimization problem solved by means of linear programming techniques. More precisely, boundary conditions and problem constraints are expressed by linear inequalities on a column vector $\mathbf{u}$, representing the input signal (i.e the jerk) at sampling times. Hence, the minimumtime planning problem is reformulated as a feasibility test for a linear programming problem, and the minimum number of steps required to complete the given transiction can be found through a simple bisection algorithm.

The use of linear programming techniques for solving minimum-time problems for linear discrete-time systems subject to bounded inputs dates back to Zadeh [6]. Subsequently, many contributions have appeared focusing on

\footnotetext{
1 Corresponding author.

$\dagger$ This work has been supported by PRIN scientific research funds of the Italian Ministry of University and Research.
}

various improvements. For example a faster algorithm is proposed in [7]. For what concerns time-optimal control for continuous-time systems, a related result, under different hypotheses, is presented in [8]. The paper is organized as follows. Section II states the minimum-time constrained planning problem and introduces a sufficient condition for the existence of a time-optimal solution. The system discretization and the linear programming problem are presented in Section III. Section IV describes with details the bisection algorithm. Section V and VI report a few velocity planning examples and final remarks respectively.

\section{THE PROBLEM AND A SUFFICIENT CONDITION}

The problem faced in this paper is the minimum-time planning of a smooth velocity profile $v(t) \in P C^{2}\left(\left[0, t_{f}\right]\right)$, where $t_{f}$ represents the travelling minimum-time along a given path whose length is equal to $s_{f}$, respecting given velocity, acceleration, and jerk constraints. The following definition will be used along this paper.

Definition 1: A function $f: \mathbb{R} \rightarrow \mathbb{R}, t \rightarrow f(t)$ has a $P C^{2}$ continuity, or piecewise $C^{2}$-continuity, and we write $f(t) \in P C^{2}$ if

1) $f(t) \in C^{1}(\mathbb{R})$,

2) $f(t) \in C^{2}\left(\mathbb{R}-\left\{t_{1}, t_{2}, \ldots\right\}\right)$

3) $\exists \lim _{t \rightarrow t_{i}^{-}} \ddot{f}(t), \exists \lim _{t \rightarrow t_{i}^{+}} \ddot{f}(t), i=1,2, \ldots$ where $\left\{t_{1}, t_{2}, \ldots\right\}$ is a set of discontinuity instants. The posed problem can be summarized as follows

$$
\min _{v \in P C^{2}} t_{f}
$$

such that

$$
\begin{gathered}
\int_{0}^{t_{f}} v(\xi) d \xi=s_{f} \\
v(0)=v_{0}, \quad v\left(t_{f}\right)=v_{f} \\
\dot{v}(0)=a_{0}, \quad \dot{v}\left(t_{f}\right)=a_{f} \\
|v(t)| \leq v_{M} \quad \forall t \in\left[0, t_{f}\right], \\
|\dot{v}(t)| \leq a_{M} \quad \forall t \in\left[0, t_{f}\right], \\
|\ddot{v}(t)| \leq j_{M} \quad \forall t \in\left[0, t_{f}\right],
\end{gathered}
$$

where $s_{f}>0, v_{M}, a_{M}, j_{M}>0$ and $v_{0}, v_{f}, a_{0}, a_{f} \in$ $\mathbb{R}$ are given boundary conditions. For the special case of 
zero boundary conditions $\left(v_{0}=v_{f}=0, a_{0}=a_{f}=0\right)$ a closed form solution has been provided by [9]. Remark that in our context of iterative autonomous navigation, it is crucial to consider generic boundary conditions on initial and final velocities and accelerations.

The minimum-time constrained planning problem can be easily recast into a minimum-time control problem with respect to a suitable state-space system. Indeed consider the jerk $\ddot{v}(t)$ as the control input $u(t)$ of the cascade of three integrators as depicted in Figure 1.

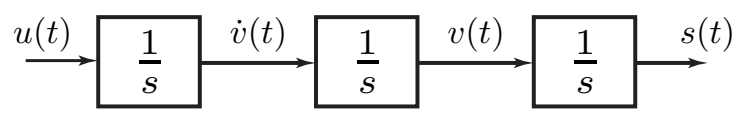

Fig. 1. The system model for velocity planning

Introduce the state $\mathbf{x}(t)$ as the following column vector

$$
\mathbf{x}(t):=\left[\begin{array}{c}
x_{1}(t) \\
x_{2}(t) \\
x_{3}(t)
\end{array}\right]:=\left[\begin{array}{c}
s(t) \\
v(t) \\
\dot{v}(t)
\end{array}\right]
$$

Then, the system equations are given by

$$
\dot{\mathbf{x}}(t)=\mathbf{A} \mathbf{x}(t)+\mathbf{B} u(t)
$$

where

$$
\mathbf{A}=\left[\begin{array}{lll}
0 & 1 & 0 \\
0 & 0 & 1 \\
0 & 0 & 0
\end{array}\right] \text { and } \mathbf{B}=\left[\begin{array}{l}
0 \\
0 \\
1
\end{array}\right]
$$

Constraints (5), (6) and (7) will be considered as two state constraints and an input bound respectively. Hence, problem (1)-(5) is equivalent to finding a time-optimal control $u^{*}(t)$ that brings system (9) from the initial state $\mathbf{x}(0)=\left[\begin{array}{lll}0 & v_{0} & a_{0}\end{array}\right]^{T}$ to the final state $\mathbf{x}\left(t_{f}^{*}\right)=\left[\begin{array}{lll}s_{f} & v_{f} & a_{f}\end{array}\right]^{T}$ in minimum time $t_{f}^{*}$, while satisfying the following constraints

$$
\begin{aligned}
& \left|x_{2}(t)\right| \leq v_{M} \quad \forall t \in\left[0, t_{f}^{*}\right], \\
& \left|x_{3}(t)\right| \leq a_{M} \quad \forall t \in\left[0, t_{f}^{*}\right],
\end{aligned}
$$

and

$$
\left|u^{*}(t)\right| \leq j_{M} \quad \forall t \in\left[0, t_{f}^{*}\right]
$$

The existence of the solution $u^{*}(t)$ of the problem (1)-(7) depends on the values of the initial state $\mathbf{x}_{0}$, the final state $\mathbf{x}_{f}$, and it also depends on the values of the constraints (11)(13). To guarantee the existence of the optimal control $u^{*}(t)$, these values must respect four sufficient conditions as stated in the following result.

Proposition 1: The minimum-time optimal control $u^{*}(t)$, solution of the problem (1)-(7), from initial state $\mathbf{x}(0)=$ $\left[\begin{array}{lll}0 & v_{0} & a_{0}\end{array}\right]^{T}$ to final state $\mathbf{x}\left(t_{f}^{*}\right)=\left[\begin{array}{lll}s_{f} & v_{f} & a_{f}\end{array}\right]^{T}$ exists if the following sufficient conditions are satisfied:

- $\left|v_{0}\right| \leq v_{M}$ and $\left|a_{0}\right| \leq a_{M}$;

- if $a_{0}>0$ :

$$
v_{0}+\frac{1}{2} \frac{a_{0}^{2}}{j_{M}} \leq v_{M}
$$

- if $a_{0}<0$ :

$$
v_{0}-\frac{1}{2} \frac{a_{0}^{2}}{j_{M}} \geq-v_{M}
$$

- if $a_{f}<0$ :

$$
v_{f}+\frac{1}{2} \frac{a_{f}^{2}}{j_{M}} \leq v_{M}
$$

- if $a_{f}>0$ :

$$
v_{f}-\frac{1}{2} \frac{a_{f}^{2}}{j_{M}} \geq-v_{M} .
$$

Proof: Consider the case $a_{0}>0$; if condition (14) on initial state $\mathbf{x}_{0}$ is true, it is possible to apply a control function $u(t)=-j_{M}$ which brings the acceleration $x_{3}(t)$ to zero before the velocity $x_{2}(t)$ exceeds its boundary value $v_{M}$, as depicted in Figure 2. In fact, if $u(t)=-j_{M}$ with $t \in\left[0, t_{c 1}\right]$

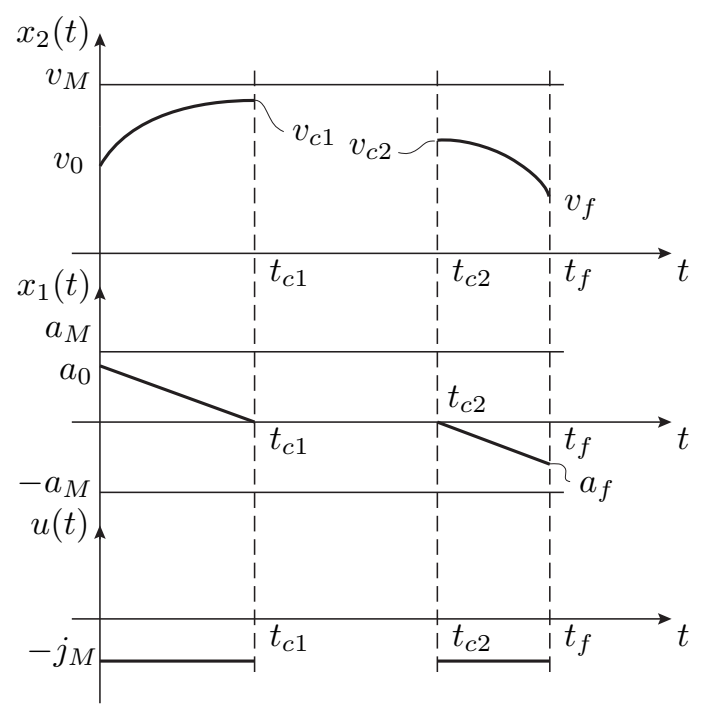

Fig. 2. Sufficient conditions on velocity/acceleration initial and final values

(where $t_{c 1}$ is the critical time where the acceleration became null) the following result is true

$$
\begin{aligned}
x_{3}(t) & =a_{0}+\int_{0}^{t_{c 1}} u(\xi) d \xi \\
& =a_{0}+\int_{0}^{t_{c 1}}\left(-j_{M}\right) d \xi \\
& =a_{0}-j_{M} t_{c 1} .
\end{aligned}
$$

But in $t=t_{c 1}$ we have $x_{3}\left(t_{c 1}\right)=0$ so it is possible to obtain the critical time value as follows

$$
t_{c 1}=\frac{a_{0}}{j_{M}} .
$$

Integrating equation (18) in $t_{c 1}$, the value of $v_{c 1}=x_{2}\left(t_{c 1}\right)$ is obtained

$$
\begin{aligned}
v_{c 1} & =v_{0}+\int_{0}^{t_{c 1}} x_{3}(\xi) d \xi \\
& =v_{0}+\int_{0}^{t_{c 1}}\left(a_{0}-j_{M} \xi\right) d \xi \\
& =v_{0}+a_{0} t_{c 1}-\frac{1}{2} j_{M} t_{c 1}^{2} .
\end{aligned}
$$


Hence, by substituting relation (18) in (20) we have

$$
v_{c 1}=v_{0}+\frac{1}{2} \frac{a_{0}^{2}}{j_{M}},
$$

then, by virtue of condition (14) we know that $v_{c 1} \leq v_{M}$ and the constraint (5) is satisfied. The same results are true for the final state value. Consider the case of $a_{f}<0$; if condition (16) is verified the velocity value $x_{2}\left(t_{c 2}\right)=v_{c 2}$ does not exceed the boundary value $v_{M}$. Hence, the final state $x\left(t_{f}\right)$ can be achieved by applying the control function $u(t)=-j_{M}$ with $t \in\left[t_{c 2}, t_{f}\right]$ as depicted in Figure 2. Now consider the time-interval $\left[t_{c 1}, t_{c 2}\right]$. It is always possible to determine a polynomial jerk function $j(t)=a_{0}+a_{1} t+$ $a_{2} t^{2}$, with $t \in\left[t_{c 1}, t_{c 2}\right]$, such that the following boundary conditions are verified:

$$
x_{3}\left(t_{c 2}\right)=0 \quad x_{2}\left(t_{c 2}\right)=v_{c 2} \quad x_{1}\left(t_{c 2}\right)=s_{f}-s_{2},
$$

where $s_{2}=\int_{t_{c 2}}^{t_{f}} x_{2}(\xi) d \xi$, while satisfying the constraints (11)- (13). This is true because the transition timeinterval $\left[t_{c 1}, t_{c 2}\right]$ can be chosen arbitrary large. The sufficient conditions (15) and (17) can be easily proved in the same way.

The conditions introduced in Proposition 1 are sufficient but not necessary. In fact, for a minimum time $t_{f}^{*}$ sufficiently small, the conditions (14)-(17) can be violated, while still satisfying the constraints (11)-(13).

\section{AN APPROXIMATED SOLUTION USING DISCRETIZATION}

This section shows how to find a numerically approximated solution of problem (1)-(7) by discretization of system (9). The technique that will be introduced, exploits the results presented by Consolini and Piazzi in [10], which shows that, given a continuous-time system, an approximated optimal control can be found through the following procedure:

1) find the discretized system with sampling period $T_{s}$;

2) find the optimal input sequence $u^{*}(k)$;

3) use for the continuous-time system the input function $u(t)$ obtained from the discrete-time sequence with a zero-order hold

$$
u(t)=u_{T_{s}}^{*}\left(\left\lfloor\frac{t}{T_{s}}\right\rfloor\right),
$$

where $T_{s} \in \mathbb{R}$ is the sampling period and $\forall x \in \mathbb{R}$ $\lfloor x\rfloor=\max \{z \in \mathbb{Z}: z \leq x\}$ denotes the integer part of $x$.

As shown in [10], when $T_{s} \rightarrow 0$ the approximated solution converges to the optimal continuous-time solution.

The optimal discrete-time control sequence $u^{*}(t)$ can be found by means of linear programming. In fact, in the discrete-time case, the constraints (11)-(13) can be represented as linear inequalities and the minimum number of steps needed for the requested transition can be found through a sequence of feasibility tests of a linear programming problem.
The matrices of the equivalent discrete-time system are the following ones:

$$
\mathbf{A}_{d}=e^{\mathbf{A} T_{s}}=\left[\begin{array}{ccc}
1 & T_{s} & \frac{1}{2} T_{s}^{2} \\
0 & 1 & T_{s} \\
0 & 0 & 1
\end{array}\right]
$$

and

$$
\mathbf{B}_{d}=\mathbf{f}\left(\mathbf{A}, T_{s}\right) \mathbf{B}=\left(\int_{0}^{T_{s}} e^{\mathbf{A} \tau} d \tau\right) \mathbf{B}=\left[\begin{array}{c}
\frac{1}{6} T_{s}^{3} \\
\frac{1}{2} T_{s}^{2} \\
T_{s}
\end{array}\right],
$$

where $T_{s}$ is the sampling period. Then, the discrete-time system is

$$
\mathbf{x}(k+1)=\mathbf{A}_{d} \mathbf{x}(k)+\mathbf{B}_{d} \mathbf{u}(k),
$$

whose solution is given by

$$
\mathbf{x}(k)=\mathbf{A}_{d}^{k} \mathbf{x}_{0}+\sum_{j=0}^{k-1} \mathbf{A}_{d}^{k-1-j} \mathbf{B}_{d} \mathbf{u}(j)
$$

where

$$
\mathbf{x}(k)=\left[\begin{array}{c}
x_{1}(k) \\
x_{2}(k) \\
x_{3}(k)
\end{array}\right]
$$

Define the control vector $\mathbf{u} \in \mathbb{R}^{k_{f}}$ as follows

$$
\mathbf{u}=\left[\begin{array}{c}
u(0) \\
u(1) \\
\vdots \\
u\left(k_{f}-1\right)
\end{array}\right]
$$

from (13) it follows that it must be

$$
-u_{M} \cdot \mathbf{1}_{k_{f}} \leq \mathbf{u} \leq u_{M} \cdot \mathbf{1}_{k_{f}}
$$

where $\mathbf{1}_{k_{f}}$ denotes the $k_{f}$-dimensional vector whose components are all equal to 1 . The velocity constraint for discretetime system is given by

$$
-v_{M} \leq x_{2}(k) \leq v_{M}, \quad \text { with } \quad k=0, \ldots, k_{f}-1 .
$$

From equation (27), velocity sequence $x_{2}(k)$ can be written as follows

$$
\begin{aligned}
x_{2}(k) & =\mathbf{C}_{1} \mathbf{x}(k) \\
& =\mathbf{C}_{1}\left[\mathbf{A}_{d}^{k} \mathbf{x}_{0}+\sum_{j=0}^{k-1} \mathbf{A}_{d}^{k-1-j} \mathbf{B}_{d} \mathbf{u}(j)\right] \\
& =\mathbf{C}_{1} \mathbf{A}_{d}^{k} \mathbf{x}_{0}+\sum_{j=0}^{k-1} \mathbf{C}_{1} \mathbf{A}_{d}^{k-1-j} \mathbf{B}_{d} \mathbf{u}(j),
\end{aligned}
$$

where

$$
\mathbf{C}_{1}=\left[\begin{array}{lll}
0 & 1 & 0
\end{array}\right] .
$$

By substituting (32) in (31), the following relation is obtained

$$
-v_{M}-\mathbf{C}_{1} \mathbf{A}_{d}^{k} \mathbf{x}_{0} \leq \sum_{j=0}^{k-1} \mathbf{C}_{1} \mathbf{A}_{d}^{k-1-j} \mathbf{B}_{d} \mathbf{u}(j) \leq v_{M}-\mathbf{C}_{1} \mathbf{A}_{d}^{k} \mathbf{x}_{0}
$$


with $k=0, \ldots, k_{f}-1$. Set $\mathbf{v}_{c}=v_{M} \cdot \mathbf{1}_{f}$, then the inequality on velocity constraint (31) can be written as follows

$$
-\mathbf{v}_{c}-\mathbf{G}_{1} \leq \mathbf{H}_{1} \mathbf{u} \leq \mathbf{v}_{c}-\mathbf{G}_{1}
$$

where $\mathbf{G}_{1} \in \mathbb{R}^{k_{f}}$ and $\mathbf{H}_{1} \in \mathbb{R}^{k_{f} \times k_{f}}$ are given by

$$
\mathbf{G}_{1}=\left[\begin{array}{c}
\mathbf{C}_{1} \mathbf{x}_{0} \\
\mathbf{C}_{1} \mathbf{A}_{d} \mathbf{x}_{0} \\
\mathbf{C}_{1} \mathbf{A}_{d}^{2} \mathbf{x}_{0} \\
\vdots \\
\mathbf{C}_{1} \mathbf{A}_{d}^{k_{f}-1} \mathbf{x}_{0}
\end{array}\right]
$$

and

$$
\mathbf{H}_{1}=\left[\begin{array}{cccc}
\mathbf{C}_{1} \mathbf{B}_{d} & \mathrm{O} & \cdots & \mathrm{O} \\
\mathbf{C}_{1} \mathbf{A}_{d} \mathbf{B}_{d} & \ddots & \ddots & \mathrm{O} \\
\mathbf{C}_{1} \mathbf{A}_{d}^{2} \mathbf{B}_{d} & \ddots & \ddots & \mathrm{O} \\
\vdots & \ddots & \ddots & \vdots \\
\mathbf{C}_{1} \mathbf{A}_{d}^{k_{f}-1} \mathbf{B}_{d} & \cdots & \cdots & \mathbf{C}_{1} \mathbf{B}_{d}
\end{array}\right]
$$

The acceleration constraint for discrete-time system (26) is given by

$$
-a_{M} \leq x_{3}(k) \leq a_{M}, \quad \text { with } \quad k=0, \ldots, k_{f}-1 .
$$

Set $\mathbf{a}_{c}=a_{M} \cdot \mathbf{1}_{f}$ and

$$
\mathbf{C}_{2}=\left[\begin{array}{lll}
0 & 0 & 1
\end{array}\right],
$$

then constraint (38) is written as

$$
-\mathbf{a}_{c}-\mathbf{G}_{2} \leq \mathbf{H}_{2} \mathbf{u} \leq \mathbf{a}_{c}-\mathbf{G}_{2},
$$

where $\mathbf{G}_{2} \in \mathbb{R}^{k_{f}}$ and $\mathbf{H}_{2} \in \mathbb{R}^{k_{f} \times k_{f}}$ are given by

$$
\mathbf{G}_{2}=\left[\begin{array}{c}
\mathbf{C}_{2} \mathbf{x}_{0} \\
\mathbf{C}_{2} \mathbf{A}_{d} \mathbf{x}_{0} \\
\mathbf{C}_{2} \mathbf{A}_{d}^{2} \mathbf{x}_{0} \\
\vdots \\
\mathbf{C}_{2} \mathbf{A}_{d}^{k_{f}-1} \mathbf{x}_{0}
\end{array}\right]
$$

and

$$
\mathbf{H}_{2}=\left[\begin{array}{cccc}
\mathbf{C}_{2} \mathbf{B}_{d} & \mathrm{O} & \cdots & \mathrm{O} \\
\mathbf{C}_{2} \mathbf{A}_{d} \mathbf{B}_{d} & \ddots & \ddots & \mathrm{O} \\
\mathbf{C}_{2} \mathbf{A}_{d}^{2} \mathbf{B}_{d} & \ddots & \ddots & \mathrm{O} \\
\vdots & \ddots & \ddots & \vdots \\
\mathbf{C}_{2} \mathbf{A}_{d}^{k_{f}-1} \mathbf{B}_{d} & \cdots & \cdots & \mathbf{C}_{2} \mathbf{B}_{d}
\end{array}\right]
$$

The interpolation condition on final state can be written as follows

$$
\mathbf{x}_{f}=\mathbf{x}\left(k_{f}\right)=\left[\begin{array}{c}
x_{1}\left(k_{f}\right) \\
x_{2}\left(k_{f}\right) \\
x_{3}\left(k_{f}\right)
\end{array}\right]=\left[\begin{array}{c}
s_{f} \\
v_{f} \\
a_{f}
\end{array}\right] .
$$

From equation (27) we have

$$
\mathbf{x}_{f}=\mathbf{A}_{d}^{k_{f}} \mathbf{x}_{0}+\sum_{j=0}^{k_{f}-1} \mathbf{A}_{d}^{k_{f}-1-j} \mathbf{B}_{d} \mathbf{u}(j)
$$

then, by substituting equation (44) in (43) we obtain the final state interpolation condition as follows

$$
\mathbf{H}_{e q} \mathbf{u}=\mathbf{x}_{f}-\mathbf{A}_{d}^{k_{f}} \mathbf{x}_{0}
$$

where $\mathbf{H}_{e q} \in \mathbb{R}^{3 \times k_{f}}$ is given by

$$
\mathbf{H}_{e q}=\left[\begin{array}{lllll}
\mathbf{A}_{d}^{k_{f}-1} \mathbf{B}_{d} & \mathbf{A}_{d}^{k_{f}-2} \mathbf{B}_{d} & \cdots & \mathbf{B}_{d}
\end{array}\right] .
$$

In conclusion given a number of steps $k_{f}$, there exists an input vector $\mathbf{u}$ for which the constraints on velocity, acceleration and jerk, and the final interpolation condition are satisfied if and only if the following linear programming problem is feasible

$$
\left\{\begin{array}{l}
-u_{M} \cdot \mathbf{1}_{k_{f}} \leq \mathbf{u} \leq u_{M} \cdot \mathbf{1}_{k_{f}} \\
-\mathbf{v}_{c}-\mathbf{G}_{1} \leq \mathbf{H}_{1} \mathbf{u} \leq \mathbf{v}_{c}-\mathbf{G}_{1} \\
-\mathbf{a}_{c}-\mathbf{G}_{2} \leq \mathbf{H}_{2} \mathbf{u} \leq \mathbf{a}_{c}-\mathbf{G}_{2} \\
\mathbf{H}_{e q} \mathbf{u}=\mathbf{x}_{f}-\mathbf{A}_{d}^{k_{f}} \mathbf{x}_{0} .
\end{array}\right.
$$

\section{THE BISECTION ALGORITHM}

The minimum number of steps $k_{f}^{*}$ and the associated optimal discrete-time control sequence $u^{*}(k)$, with $k=0, \ldots, k_{f}^{*}-1$, can be determinated by means of a sequence of linear programming feasibility tests, defined by (47), through a simple bisection algorithm. The Minimum-Time Control algorithm (MTC) is summarized as follows:

$$
\begin{aligned}
& \text { begin } \\
& k_{f} \leftarrow 1 ; \\
& l \leftarrow 0 ; \\
& \text { while } \sim \boldsymbol{L P P} \text { do } \\
& l \leftarrow k_{f} \\
& k_{f} \leftarrow 2 k_{f} \\
& \text { end } \\
& h \leftarrow k_{f} ; \\
& \text { while } h-l>1 \text { do } \\
& k_{f} \leftarrow\left\lfloor\frac{h+l}{2}\right\rfloor ; \\
& \text { if } \sim \boldsymbol{L P P} \text { then } \\
& l \leftarrow k_{f} ; \\
& \text { else } \\
& h \leftarrow k_{f} ; \\
& \text { end } \\
& k_{f}^{*} \leftarrow h ; \\
& u^{*}(k) \leftarrow \mathbf{u} ; \\
& \text { end } \quad
\end{aligned}
$$

In MTC algorithm $\boldsymbol{L P P}$ denotes a linear programming procedure that solves problem (47), which, if a feasible solution exists, returns the solution sequence $\mathbf{u}$ and the number of steps $k$; if the problem is feasible it also returns a Boolean true value.

The algorithm performances strongly depend on the used sampling time: by reducing $T_{s}$, which means sampling the continuous-time system with an higher frequency, the dimension of the resulting linear programming problem increases, thus causing an increment of the total computational time. 
Considering the computational complexity, Karmarkar has shown in [11] that a linear programming problem can be solved by means of an interior-point algorithm with running time proportional to $n^{3.5}$, where $n$ is the number of inequalities. In our case this would means that each feasibility test would require a time proportional to $n_{s}^{3.5}$, where $n_{s}$ is the total number of samples. The complexity of the bisection search, with respect to the minimum number of samples, is given by $O\left(\log n_{s}\right)$, therefore the total complexity of the proposed algorithm is given by $O\left(n_{s}^{3.5} \log n_{s}\right)$. For more details on the algorithm complexity see [12].

\section{EXAMPLES}

Example 1: consider the following interpolation conditions and constraints:

- initial state

$$
\mathbf{x}_{0}:=\left[\begin{array}{l}
s_{0} \\
v_{0} \\
a_{0}
\end{array}\right]:=\left[\begin{array}{l}
0 \\
0 \\
0
\end{array}\right]
$$

- final state

$$
\mathbf{x}_{f}:=\left[\begin{array}{c}
s_{f} \\
v_{f} \\
a_{f}
\end{array}\right]:=\left[\begin{array}{l}
2 \\
0 \\
0
\end{array}\right]
$$

- problem constraints

$$
v_{M}=0,65[\mathrm{~m} / \mathrm{s}] \quad a_{M}=0.5\left[\mathrm{~m} / \mathrm{s}^{2}\right] \quad j_{M}=0.5\left[\mathrm{~m} / \mathrm{s}^{3}\right]
$$

The jerk, acceleration, velocity and space profiles, obtained by means of the MTC algorithm, are depicted in Figure 3.

Example 2: consider the following problem:

- initial state

$$
\mathbf{x}_{0}:=\left[\begin{array}{l}
s_{0} \\
v_{0} \\
a_{0}
\end{array}\right]:=\left[\begin{array}{l}
0 \\
0 \\
0
\end{array}\right]
$$

- final state

$$
\mathbf{x}_{f}:=\left[\begin{array}{c}
s_{f} \\
v_{f} \\
a_{f}
\end{array}\right]:=\left[\begin{array}{c}
2 \\
1 \\
0,25
\end{array}\right]
$$

- problem constraints

$$
v_{M}=1,5[\mathrm{~m} / \mathrm{s}] \quad a_{M}=0.6\left[\mathrm{~m} / \mathrm{s}^{2}\right] \quad j_{M}=0.5\left[\mathrm{~m} / \mathrm{s}^{3}\right]
$$

The jerk, acceleration, velocity and space profiles, obtained in this case, are depicted in Figure 4.

Example 3: the problem data are given by:

- initial state

$$
\mathbf{x}_{0}:=\left[\begin{array}{c}
s_{0} \\
v_{0} \\
a_{0}
\end{array}\right]:=\left[\begin{array}{c}
0 \\
1 \\
-0.5
\end{array}\right]
$$

- final state

$$
\mathbf{x}_{f}:=\left[\begin{array}{c}
s_{f} \\
v_{f} \\
a_{f}
\end{array}\right]:=\left[\begin{array}{c}
2,167 \\
0,5 \\
0,5
\end{array}\right]
$$
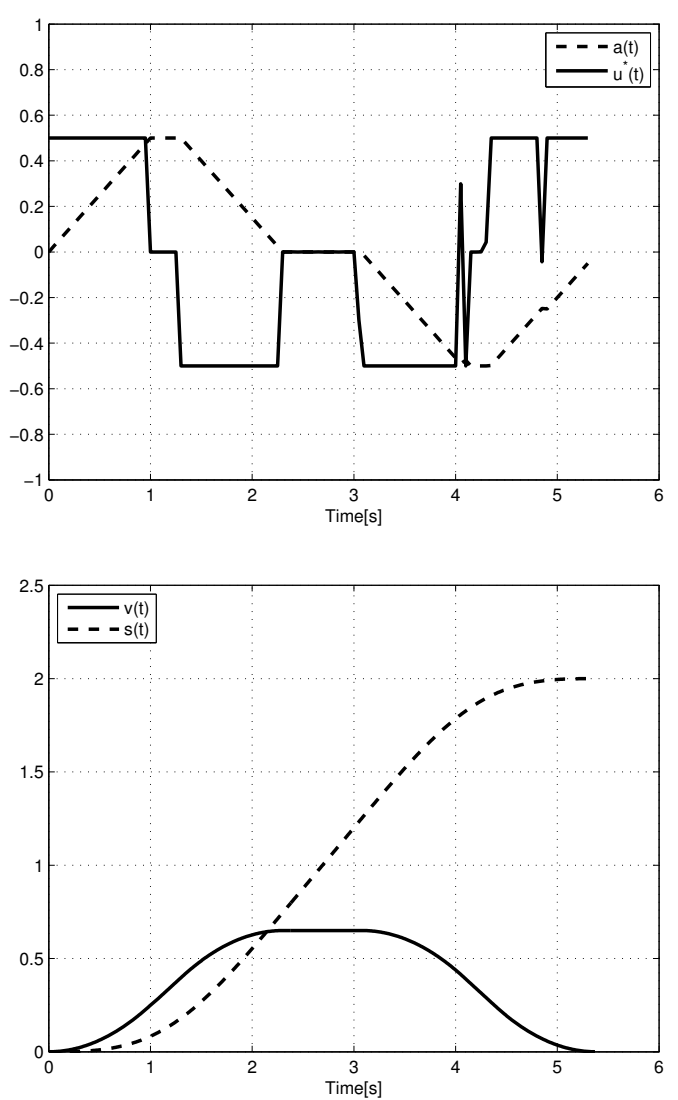

Fig. 3. Optimal solutions for Example 1

- problem constraints

$$
v_{M}=1[\mathrm{~m} / \mathrm{s}] \quad a_{M}=0.5\left[\mathrm{~m} / \mathrm{s}^{2}\right] \quad j_{M}=0.5\left[\mathrm{~m} / \mathrm{s}^{3}\right]
$$

Figure 5 shows optimal solution obtained by means of the MTC algorithm.

\section{CONCLUSION}

This paper has proposed a method for velocity planning considering velocity, acceleration and jerk constraints. A sufficient condition on initial and final states was introduced. The minimum-time planning problem is solved by discretization of the continuous-time system, formulating an equivalent discrete-time optimization problem solved by linear programming techniques. The MTC algorithm, which determines an approximation of the minimum-time solution, is well suited to be adopted into a supervisory architecture for iterative steering navigation.

\section{REFERENCES}

[1] S. M. LaValle, Planning Algorithms. Cambridge, U.K.: Cambridge University Press, 2006.

[2] B. Siciliano and O. Khatib, Eds., Springer Handbook of Robotics. Springer, 2008.

[3] K. Kant and S. Zucker, "Toward efficient trajectory planning: the pathvelocity decomposition," Int. J. of Robotics Research, vol. 5, no. 3, pp. 72-89, 1986. 

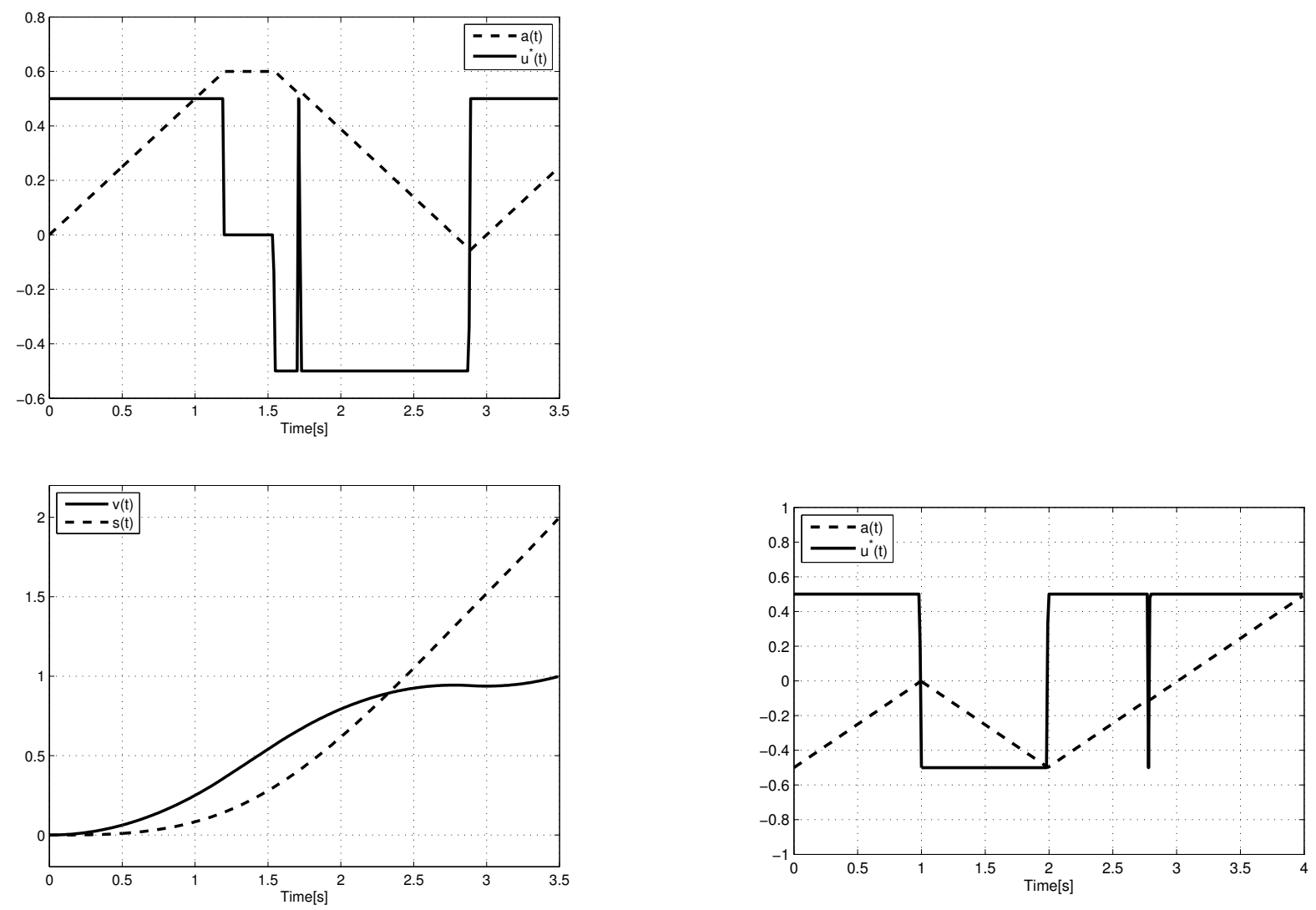

Fig. 4. Optimal solutions for Example 2

[4] P. Lucibello and G. Oriolo, "Robust stabilization via iterative state steering with an application to chained-form systems," Automatica, vol. 37, no. 1, pp. 71-79, January 2001.

[5] C. Guarino Lo Bianco, A. Piazzi, and M. Romano, "Smooth motion generation for unicycle mobile robots via dynamic path inversion," IEEE Trans. on Robotics, vol. 20, no. 5, pp. 884-891, Oct. 2004.

[6] L. A. Zadeh, "On optimal control and linear programming," IRE Transactions on Automatic Control, vol. 7, no. 4, pp. 45-46, 1962.

[7] G. Bashein, "A simplex algorithm for on-line computation of optimal controls," IEEE Transactions on Automatic Control, vol. 16, no. 5, pp. 479-482, 1971.

[8] S.-J. Kim, D.-S. Choi, and I.-J. Ha, "A comparison principle for stateconstrained differential inequalities and its application to time-optimal control," IEEE Transactions on Automatic Control, vol. 50, no. 7, pp. 967-983, July 2005.

[9] R. D. Peters, "Ideal lift kinematics: Complete equations for plotting optimum motion," Proceedings of ELEVCON95 (The International Association of Elevator Engineers), 1995, republished by Elevator World, April 1996 and by Elevatori, May/June 1996.

[10] L. Consolini and A. Piazzi, "Generalized bang-bang control for feedforward constrained regulation," Proceedings of the 45th IEEE Conference on Decision and Control, pp. 893-898, 2006.

[11] N. Karmakar, "A new polynomial-time algorithm for linear programming," Report. AT\&T Bell Laboratories, 1984.

[12] L. Consolini, O. Gerelli, C. Guarino Lo Bianco, and A. Piazzi, "Flexible joints control: A minimum-time feed-forward technique," Mechatronics, Elsevier, vol. 19, pp. 348-356, Oct. 2008 